\title{
El periodismo de paz como paradigma de comunicación para el cambio social: características, dimensiones y obstáculos
}

\author{
Eva Espinar Ruiz y Maria Isabel HernÁNDez SÁNChez*
}

\author{
Propuesto: 6 de marzo de 2012 \\ Evaluado: 12 de abril de 2012 \\ Aceptado: 25 de abril de 2012 \\ (Abstracts y palabras clave al final del texto)
}

\section{INTRODUCCIÓN}

Los medios de comunicación se han convertido hoy día en la principal fuente de información sobre la realidad. En consecuencia, ejercen una influencia fundamental en la comprensión que la audiencia tiene de tal realidad e, indirectamente, en su actitud hacia la misma (Curran, 2005: 168). Es más, es tal la relevancia del "universo mediático", que podemos afirmar que éste incide no sólo en la manera "de percibir el mundo", sino también en "su funcionamiento económico, cultural y político" (Penalva, 2002: 396). De esta forma, el proceder de los medios de comunicación en un contexto social dado no es inocuo; los medios no son meros observadores de los procesos sociales sino participantes en los mismos. Este rol es especialmente relevante en el caso concreto de guerras o conflictos armados, donde los medios participan, inevitablemente, en el ciclo del conflicto. Tal y como plantea Hackett (2007: 48), en tiempo de guerra, los medios actúan, simultáneamente, como fuentes de información, combatientes, armas, objetivos y campo de batalla.

En concreto, Vicenç Fisas (1998: 71) destaca el papel de los medios en la configuración de "actitudes sociales en relación a los conflictos y su transformación". Así, los medios pueden fomentar "comportamientos agresivos, justificar acciones bélicas, formar estereotipos, imágenes del enemigo y demonizaciones". Quizá el más conocido de entre los múltiples ejemplos documentados sea el caso ruandés, paradigma de los denominados "medios del odio" (Thompson, 2007; Frère, 2007), si bien los ejemplos son abundantes, como muestran las investigaciones en torno a la influencia mediática en la propaganda de guerra (Tortosa, 2003).

Sin embargo, los medios también pueden "despertar compasión, misericordia y caridad, estimular exigencias de actuación, cambios de políticas o romper distancias" (Fisas, 1998: 72). Podemos encontrar, así, ejemplos en los que los medios han

* Departamento de Sociología II, Universidad de Alicante. 
puesto en práctica su capacidad para denunciar violaciones de los derechos humanos o para disminuir el nivel de tensión en situaciones de conflicto y promover procesos de paz. Entre estas tendencias, destaca la puesta en práctica del periodismo de paz, un paradigma relativamente reciente (su origen suele situarse en los años 70 del siglo XX) que hunde sus raíces en la investigación para la paz y se fundamenta en la aplicación de la teoría y el análisis de conflictos al ámbito de la comunicación (Lynch, 2008; Peleg, 2006). Precisamente, el objetivo de este artículo es recoger las principales características de lo que se ha denominado periodismo de paz, cuyos rasgos esenciales lo distinguen del paradigma periodístico dominante en la actualidad. De esta forma, se destaca, en los siguientes epígrafes, sus dimensiones y aportaciones prácticas, analíticas y teóricas; así como las barreras y obstáculos que dificultan, hoy día, su desarrollo y aplicación.

\section{TRATAMIENTO MEDIÁTICO DE LOS CONFLICTOS: PARADIGMAS PERIODÍSTICOS}

El conflicto, la violencia y, en definitiva, las malas noticias constituyen "la materia prima por excelencia de las informaciones que ofrecen los medios de comunicación" (Giró, 2007: 199). En el contexto de guerras y conflictos armados esta preferencia parece acentuarse. Así, por ejemplo, el Institute for Economics and Peace (IEP, 2010), tras el análisis de la cobertura mediática sobre conflictos armados en 15 países distintos, destaca la presencia de un interés desproporcionado por la violencia y un desinterés manifiesto por la paz. Sólo el 1,6 por ciento del material analizado se corresponde con historias positivas relativas a avances en la transformación del conflicto. A estas mismas conclusiones llegaba, ya en los años 60, uno de los promotores del periodismo de paz, el investigador noruego Johan Galtung. Galtung y Ruge (1965), a partir del análisis de la cobertura sobre una serie conflictos en cuatro diarios noruegos, plantean: primero, que los actos de violencia se convierten en acontecimientos noticiables en sí mismos y, segundo, que al tratar el tema de la violencia los medios siempre obvian un factor, la paz.

Galtung (1998a) plantea que existen dos modelos o paradigmas periodísticos a la hora de abordar los conflictos: el dominante o periodismo de guerra y el alternativo o periodismo de paz (las características principales de ambos enfoques, tal y como las resume el investigador noruego, pueden consultarse en el cuadro 1). En primer lugar, el paradigma hegemónico o periodismo de guerra reúne los siguientes rasgos (Galtung, 2002):

1. Orientación hacia la guerra y la violencia, con una clara confusión de términos entre conflicto y violencia. Desde la perspectiva de la investigación para la paz se plantea la necesaria distinción entre conflicto y violencia. El conflicto no es negativo en sí mismo, sino que debe ser entendido como un elemento consustancial a las relaciones humanas, un proceso en el que confluyen necesidades y objetivos en apariencia incompatibles entre sí y que puede evolucionar hasta manifestarse de forma violenta (Fisas, 1998). En palabras de 
Galtung (2000: 162): “Creo que existe una incapacidad [entre los periodistas] para distinguir entre conflicto y violencia. Si tienes esa incapacidad no vas a detectar el conflicto que provoca esa violencia, no tienes las herramientas conceptuales".

2. Fuertemente influido por la propaganda. Tal y como plantean Lee y Maslog (2005: 311), el periodismo de guerra suele identificarse con un bando o, en todo caso, con la defensa de los intereses nacionales. De igual forma, tiende a privilegiar, sistemáticamente, a las fuerzas políticas y económicas dominantes (Hackett, 2007: 49).

3. Se fundamenta en la opinión de las élites, que se convierten en la principal fuente de información. Los medios, en la búsqueda de una supuesta objetividad, acuden a las fuentes consideradas prestigiosas, es decir: las élites y fuentes oficiales, que, terminan, así, influyendo en la agenda mediática (Lynch y McGoldrick, 2005: 67).

4. Protagonismo de una perspectiva de juego de suma cero, donde un bando pierde y el otro gana. Los conflictos se simplifican, reduciendo las partes implicadas a pocos bandos enmarcados en un proceso en el que "lo que gana una parte es lo que pierde la otra" (Hackett, 2010: 183).

El periodismo de guerra asume los criterios tradicionales que convierten un hecho en noticiable, al basar sus contenidos en eventos concretos, limitados en el tiempo, descontextualizados, recientes y fácilmente explicables (Hackett, 2010: 185; Lee y Maslog, 2005: 311). De esta forma, es la violencia física directa la que centra su interés, mientras son desconsiderados otros efectos no tan reproducibles en imágenes. El resultado, independientemente de la intención inicial de los profesionales, es que esta forma de periodismo contribuye a exacerbar el conflicto, a la creación de bandos irreconciliables y a ocultar los procesos y propuestas de paz que puedan estar desarrollándose (Hackett, 2007: 49).

Frente a la práctica dominante, Galtung sitúa el paradigma del periodismo de paz, orientado hacia la transformación del conflicto, que atiende a la voz de las víctimas e interpreta la paz como un proceso en el que todas las partes han de estar involucradas y recibir beneficios. Los periodistas que se encuadran en esta perspectiva informan sobre las iniciativas de paz existentes, aportan información que contextualiza los conflictos y consideran los efectos no directamente visibles de los mismos, así como sus causas estructurales y culturales. En definitiva, se trata de un periodismo implicado en los procesos de resolución del conflicto, reconstrucción y reconciliación (Galtung 1998b), que resalta los elementos compartidos entre los actores y sectores implicados y no únicamente las diferencias. El periodismo de paz supone, así, un desafío al periodismo de guerra, en la medida en que centra su foco de atención en la contextualización y los procesos de largo plazo y, especialmente, en la diversificación de temas y fuentes de información, buscando de forma activa aquellas voces que representan opciones de resolución pacífica del conflicto (Hackett, 2010: 185). En el siguiente apartado se detallan, en mayor medida, las características y dimensiones que configuran el paradigma alternativo del periodismo de paz. 
Cuadro 1.

Características del periodismo de paz vs periodismo de guerra

\begin{tabular}{|c|c|}
\hline PERIODISMO DE PAZ/CONFLICTO & PERIODISMO DE GUERRA/VIOLENCIA \\
\hline $\begin{array}{l}\text { I.ORIENTADO HACIA LA PAZ/CONFLICTO } \\
\text { - Explorar la formación del conflicto, actores, } \\
\text { objetivos, cuestiones, perspectiva gano/ganas } \\
\text { - Espacio abierto, tiempo abierto; causas y } \\
\text { desenlaces en cualquier parte, también en } \\
\text { la historia y la cultura } \\
\text { - Hacer los conflictos transparentes } \\
\text { - Dar voz a todas las partes, empatía } \\
\text { - Ver el conflicto/guerra como un problema, } \\
\text { - Hutención a la creatividad } \\
\text { - Proactivo: prevenir la violencia/guerra } \\
\text { - Atención en los efectos invisibles de la } \\
\text { violencia: trauma, daños estructurales y } \\
\text { culturales, etc. }\end{array}$ & $\begin{array}{l}\text { II. ORIENTADO HACIA LA GUERRA/VIOLENCIA } \\
\text { - Atención centrada en el escenario del conflicto, dos } \\
\text { partes, un objetivo (ganar, la victoria) } \\
\text { - Espacio cerrado, tiempo cerrado; causas y salidas en } \\
\text { el escenario, quién lanzó la primera piedra } \\
\text { - Hacer las guerras opacas, secretas } \\
\text { - "Nosotros-ellos", propaganda, voz al nosotros } \\
\text { - "Ellos" como el problema, quién predomina } \\
\text { - Deshumanización de los "otros" } \\
\text { - Reactivo: esperar a la violencia } \\
\text { - Atención sólo sobre los efectos visibles de la vio- } \\
\text { lencia (muertos, heridos, daño material)" }\end{array}$ \\
\hline $\begin{array}{l}\text { II. ORIENTADO A LA VERDAD } \\
\text { - Exponer las mentiras de todas las partes, } \\
\text { descubrir lo que se pretende ocultar }\end{array}$ & $\begin{array}{l}\text { II. ORIENTADO A LA PROPAGANDA } \\
\text { - Exposición de "sus" mentiras. Apoyo a "nuestras" } \\
\text { mentiras/encubrimientos }\end{array}$ \\
\hline $\begin{array}{l}\text { III.ORIENTADO A LA SOCIEDAD CIVIL } \\
\text { - A tención al sufrimiento, dar voz a los sin } \\
\text { voz, mujeres, niños, ancianos } \\
\text { - Identificar a todos los "malhechores" } \\
\text { - Atención sobre los que promueven la paz }\end{array}$ & $\begin{array}{l}\text { III. ORIENTADO A LA ÉLITE } \\
\text { - Atención sobre "nuestro" sufrimiento, ser sus títeres } \\
\text { - Identificar a "sus malhechores" } \\
\text { •. Atención sobre las acciones de paz de las élites. }\end{array}$ \\
\hline $\begin{array}{l}\text { IV. ORIENTADO HACIA LA SOLUCIÓN } \\
\text { - Paz= no violencia + creatividad } \\
\text { - Destacar las iniciativas de paz } \\
\text { - Atención en la estructura y cultura, la socie- } \\
\text { dad pacífica } \\
\text { - Consecuencias: resolución, reconstrucción, } \\
\text { reconciliación }\end{array}$ & $\begin{array}{l}\text { IV. ORIENTADO HACIA LA VICTORIA } \\
\text { - Paz= victoria + alto el fuego } \\
\text { - Ocultar las iniciativas de paz hasta tener la victo- } \\
\text { ria al alcance } \\
\text { - Atención sobre el tratado, institución de la socie- } \\
\text { dad controlada } \\
\text { - Abandono hasta otro conflicto, atención si hay un } \\
\text { nuevo estallido del conflicto }\end{array}$ \\
\hline
\end{tabular}

Fuente: traducción propia a partir de J. Galtung (2007)

\section{EL PERIODISMO DE PAZ: CARACTERÍSTICAS Y DIMENSIONES}

A pesar de su relativa novedad, un número cada vez mayor de autores (Hackett 2006 y 2007; Irvan, 2006; Kempf, 2007a; Lynch y McGoldrick, 2005; Ottosen, 2007; Peleg 2006 y 2007; Tehranian, 2002) está tratando de dotar de contenido teó- 
rico, académico y profesional al periodismo de paz. En este sentido, destaca el trabajo, fundamentalmente desde un punto de vista práctico, de Jake Lynch y Annabel McGoldrick, ambos discípulos de Johan Galtung. Estos autores definen el periodismo de paz como un método más ajustado y preciso de enmarcar las historias, que pone en práctica los preceptos del análisis de conflictos y de la investigación para la paz (Lynch y McGoldrick, 2005):

Así, por ejemplo, entre otros conceptos, resulta de especial interés el triángulo de las violencias desarrollado por Johan Galtung (1990). En él se distinguen tres tipos de violencia estrechamente relacionadas entre sí: la directa, referida a la violencia física que causa daños visibles (atentados, agresiones, asesinatos, destrozos materiales, torturas, etc.); la estructural, aquel tipo de violencia que forma parte de la estructura social e impide la satisfacción de las necesidades básicas (desigualdades, racismo, pobreza, hambre, esclavitud, sexismo, etc.); y la cultural, relacionada con todos los aspectos del ámbito simbólico (religión, cultura, lengua, arte, ciencias, etc.) que se pueden emplear para justificar o legitimar las violencias estructurales o directas. La aplicación de este triángulo en el análisis de los discursos mediáticos sobre los conflictos, permite observar una sobrerrepresentación de la violencia directa y una escasa atención a las otras dos formas de violencia, la estructural y la cultural (Penalva, 2002; Giró, 2007).

Esta tendencia a identificar el conflicto con la violencia directa convierte a los propios medios en agentes culturalmente violentos. Independientemente de la intencionalidad o la voluntad con que se escriben los textos, el hecho de que en el discurso sobre el conflicto, éste se presente en clave de enfrentamientos entre partes, alteración del orden público o cualquier otro tipo de disrupción del devenir social, ilustrado con imágenes de actos violentos aparentemente espontáneos e inconexos y sin mayores argumentaciones estructurales, encaja en una espiral de acción-reacción en la que la respuesta o solución al conflicto/violencia es la represión/violencia y ésta a su vez, genera más violencia. Así, el fin del conflicto se asocia con un concepto de seguridad o "paz social" que, en términos teóricos, se corresponde con la idea de "paz negativa", lejos de la "paz positiva" que preconizan los modelos alternativos orientados al cambio social (Lederach, 2000).

De esta forma, cuando los medios hablan de paz es preciso tener presente que lo pueden hacer desde posiciones muy diversas y opuestas entre sí. En concreto, el paradigma del periodismo de paz aboga por una idea de paz que va más allá de la mera ausencia de violencia directa, la firma de un tratado o el fin de los enfrentamientos en la calle (que podríamos denominar paz negativa). De hecho, relaciona la paz con la superación de las violencias culturales y estructurales (o paz positiva) $\mathrm{y}$, en consecuencia, otorga valor noticioso a todas las iniciativas que se promueven en esta dirección. Propone superar las concepciones dualísticas y esencialistas (el 'nosotros' contra 'ellos'), dar voz a todas las partes implicadas, exponer los efectos invisibles de la violencia y dar cobertura a los procesos de reconstrucción y reconciliación (Galtung, Lynch y McGoldrick, 2006: 41). En definitiva, y frente a las críticas procedentes del paradigma dominante y posiciones conservadores, el periodismo de paz aporta una visión más completa y realista de lo que sucede en el 
mundo (Galtung, 2002). Su relevancia, remarca Shinar, "reside en la contribución a una representación real de los temas de importancia global para los profesionales de los medios y el público en general" (2009: 8).

Una de las premisas del periodismo de paz es la aceptación de su carácter dinámico, su interdisciplinariedad, la posibilidad de acercarnos a él desde distintos campos de estudio. Así, las diferentes aportaciones realizadas desde el ámbito de la Comunicación y de los Estudios de Paz se articulan en torno a lo que podemos identificar como las tres dimensiones del periodismo de paz, cada una de las cuales responde a un tipo determinado de cuestiones (véase cuadro 2).

Cuadro 2.

Dimensiones del Periodismo de Paz

\begin{tabular}{|c|c|c|}
\hline ACADÉMICA / TEÓRICA & PRÁCTICA / PROFESIONAL & NORMATIVA / ÉTICA \\
\hline $\begin{array}{l}\text { - QUÉ } \\
\text { - Epistemología } \\
\text { - Estudios de caso }\end{array}$ & $\begin{array}{l}\text { - QUIÉN / CÓMO } \\
\text { - Individual } \\
\text { - Organizacional }\end{array}$ & $\begin{array}{l}\text { - POR QUÉ } \\
\text { - Legitimación }\end{array}$ \\
\hline
\end{tabular}

Fuente: elaboración propia

La primera de las dimensiones tiene que ver con su formulación teórica, una tarea que atañe, principalmente, a la comunidad académica. El objetivo en este ámbito es el desarrollo teórico y epistemológico del periodismo de paz, siendo el diálogo crítico entre investigadores de distintas disciplinas un elemento fundamental. Tal y como plantean Shinar y Kempf, al tratarse de un paradigma joven, es necesario realizar "esfuerzos por conceptualizar las prácticas y los valores profesionales tanto en términos operacionales como teóricos" (2007: 2). El trabajo desarrollado en esta dimensión sigue dos caminos distintos pero complementarios. Por un lado, el desarrollo teórico del periodismo de paz; por otro, en su vertiente empírica, la sistematización de una metodología adecuada para evaluar el estado actual de la cobertura de conflictos.

En la investigación empírica, una de las estrategias más empleadas ha sido el análisis de contenido (Lee y Maslog, 2005; Nassanga, 2007; Shinar, 2009; Lynch, 2009), en cuya aplicación destacan los trabajos de Maslog, Lee y Kim (2006) sobre la cobertura de la guerra de Irak y otros conflictos nacionales en la prensa asiática. Estos investigadores reconvirtieron el clásico esquema de Galtung sobre el periodismo de guerra y el periodismo de paz en un conjunto de 13 indicadores, inaugurando así una nueva etapa en la investigación sobre medios y conflictos desde la perspectiva del periodismo de paz (Shinar, 2009). Además de estas investigaciones, también se han llevado a cabo interesantes análisis desde las teorías del encuadre o framing (Fawcett, 2002; Hackett y Schroeder, 2008) o desde el análisis crítico del discurso (Giró y Jarque, 2006; Giró, 2004) y otros enfoques cualitativos (Ross, 2008).

La segunda dimensión del periodismo de paz supone su interpretación como práctica profesional, considerando aspectos operativos y respondiendo a las cuestio- 
nes de ¿quién o quiénes son los encargados de ponerlo en práctica? ¿cómo? y ¿con qué instrumentos o herramientas? En este sentido, Lynch y McGoldrick apelan directamente a editores y reporteros o periodistas como promotores del periodismo de paz cuando éstos, en su día a día, "hacen elecciones - sobre qué historias contar y cómo contarlas - que crean oportunidades para que la sociedad en su conjunto considere y valore respuestas no-violentas al conflicto" (2005: 5).

Si se define el periodismo de paz como un paradigma orientado al cambio social, es un aspecto clave que el modelo pueda materializarse, ser trasladado a las redacciones e integrado en la cultura productiva de los medios y en el bagaje del propio periodista. Ésta ha sido una de las principales y primeras preocupaciones de los fundadores del modelo. Galtung y sus discípulos, Lynch y McGoldrik, han trabajo desde el inicio para adaptar la tabla que sistematiza el paradigma (cuadro 1) en listados concretos de recomendaciones, así como en propuestas formativas para profesionales de la información. En su libro Peace Journalism, por ejemplo, Lynch y McGoldrik (2005) detallan una serie de sugerencias dirigidas a los periodistas, que incluyen tanto aquello que debería evitarse como la forma en que se han de tratar los distintos aspectos relaciones con el conflicto y el lenguaje a emplear. Por su parte, Tehranian (2002: 80) resume las principales características del periodismo de paz en "10 mandamientos", un decálogo en el que se sintetizan, en un lenguaje sencillo, los contenidos del modelo desde su dimensión práctica, tal y como puede apreciarse en el cuadro 3.

Finalmente, ha de contemplarse una tercera dimensión: la normativa o ética. Como estrategia de trabajo, el periodismo de paz supone asumir la responsabilidad de los efectos que su proceder tiene en el contexto social en el que se sitúa. Aceptar tal responsabilidad y contribuir, con ello, en los procesos de resolución de conflictos es, sin duda, una opción ética. La apuesta de este paradigma por un tipo de cobertura que revalorice y visibilice las iniciativas pacíficas y no-violentas y que, al contrario del modelo hegemónico, contribuya a desescalar los niveles de violencia, enraíza con una particular visión del papel de los medios de comunicación en la sociedad. Desde este enfoque, podemos entender el periodismo de paz como "una teoría normativa que establece que los medios de comunicación deberían jugar un rol positivo en la promoción de la paz (...) y que conlleva obligaciones para los periodistas sobre qué hacer, cómo hacerlo y por qué hacerlo" (Irvan, 2006: 34). El periodismo de paz se constituye, así, en una propuesta ética de acción mediática para la transformación y el cambio social; un "modelo normativo de cobertura del conflicto responsable y consciente, que aspira a contribuir a la construcción y el mantenimiento de la paz, y a cambiar las actitudes de propietarios, anunciantes, profesionales y audiencias de los medios" (Shinar y Kempf, 2007: 2). Esto es lo que Lynch y McGoldrick (2005) han bautizado como "la ética de la intervención".

\section{DEBILIDADES, DIFICULTADES Y OBSTÁCULOS}

Pese a los avances que, desde el punto de vista de una mejor, más completa y constructiva cobertura del conflicto, supondría la incorporación del periodismo de 
Cuadro 3

Los "10 mandamientos" del periodismo de paz

1. No reducir nunca las partes de un conflicto a dos.

2. Identificar las posiciones y los intereses de todas las partes en conflicto.

3. No caer presa de una sola fuente.

4. Desarrollar un buen sentido del escepticismo. Recordar que la información es una representación. El sesgo es inherente a la condición humana.

5. Dar voz a las víctimas y a los implicados en tareas de construcción de paz para representarlos y empoderarlos.

6. Buscar soluciones pacíficas a los problemas del conflicto pero no caer en panaceas.

7. La representación mediática del conflicto puede convertirse en parte del problema si acentúa los dualismos y odios.

8. La representación mediática del conflicto puede convertirse en parte de la solución si hace uso de las tensiones creativas presentes en todo conflicto para indagar en los aspectos compartidos y las respuestas no-violentas.

9. Respetar siempre los principios éticos profesionales de precisión, veracidad, equidad y respeto por la dignidad y los derechos humanos.

10. Trascender los propios sesgos étnicos, nacionales o ideológicos para identificar y representar a todas las partes de manera justa y precisa.

Fuente: traducido y resumido a partir de M. Tehranian (2002).

paz, el paradigma se enfrenta a una serie de límites y dificultades que pueden resumirse a través de dos cuestiones generales: por un lado, ¿es legítima la implicación periodística en el cambio social?; por otro, ¿hasta qué punto es posible, en la práctica, el periodismo de paz?

En referencia a la primera pregunta, el paradigma dominante o paradigma de guerra, suele mostrarse como la única opción posible. Éste se ampara en uno de los principios incuestionables de la profesión, el de la libertad de prensa, y se define como respetuoso con el axioma básico de la objetividad (Galtung y Vicent, 1995); ambos legado de la tradición periodística norteamericana. De esta forma, cuando el enfoque dominante es asumido como objetivo y cualquier otra opción es tildada de ideológica, los espacios para la autorreflexión por parte de los propios profesionales son reducidos. Así, Hackett (2010: 186) señala que el debate en torno a la función social de los medios es más probable que se plantee en contextos específicos, como puede ser el marcado por los cambios sociales tras periodos 
de autoritarismo o cuando la contribución mediática al recrudecimiento de conflictos ha sido claramente constatada.Ahora bien, ¿hasta qué punto es realmente objetivo el paradigma dominante? ¿A quién beneficia esta supuesta objetividad? ¿Cuáles son sus consecuencias? Según Hackett y Zhao (1998), el llamado régimen de objetividad conduce, en la práctica, a la legitimación de las ideologías y relaciones de poder preestablecidas, y a una representación del mundo parcial, sesgada, en función de unos valores e instituciones dominantes. Entre otras cuestiones, porque asumir la posibilidad positivista de informar neutralmente sobre los hechos contribuye, de hecho, a "privilegiar a personalidades sobre estructuras, estrategias políticas sobre análisis de lo político, y acontecimientos puntuales sobre procesos, condiciones o contextos a largo plazo" (Hackett, 2010: 181). Igualmente, conduce "a que determinado tipo de hechos se omitan o marginen de manera rutinaria, y a desequilibrios en las interpretaciones transmitidas al público" (Lynch y McGoldrick, 2005: 84).

Sin duda, no se trata aquí de desterrar los ideales del buen periodismo, de la objetividad entendida como el respeto a la veracidad, precisión e independencia. Es más, el periodismo de paz constituye, en este sentido, un ejemplo de buen periodismo, que asume, en todo momento, su compromiso con la verdad. En este sentido, supone una invitación al cuestionamiento de las razones y procesos que llevan a privilegiar determinados tipos de hechos y fuentes, y a prestar atención a las consecuencias de estas dinámicas en los ciclos del conflicto:

El periodismo de paz [...] se posiciona a favor de la autorreflexión por parte de los periodistas en relación con los sesgos institucionalizados por las rutinas productivas, la inevitabilidad de realizar selecciones en torno a encuadres y fuentes, la no pasividad ante las fuentes, la naturaleza intervencionista del periodismo y su potencial para convertirse en cómplice involuntario de la propaganda de guerra" (Hackett, 2010: 184).

Una cuestión paralela al debate sobre la objetividad es la relativa a las dificultades y obstáculos existentes a la hora de poner en práctica las directrices del periodismo de paz. Así, por ejemplo, el propio Galtung advierte en sus escritos de la existencia de límites estructurales y culturales que dificultan el cambio de paradigma: "No va a ser una tarea fácil dado que la mayoría de los medios -escritos o audiovisuales- así como la mayoría de las universidades, son productos del sistema moderno de Estado-nación, y reflejan la perspectiva de los poderes dominantes" (Galtung, 2006: 1). En este ámbito, y aunque la referencia a los obstáculos no está ausentes en las publicaciones sobre periodismo de paz, sí podemos hablar de una tendencia a focalizar la atención en el periodista como individuo y sus posibilidades de acción. De hecho, uno de los aspectos que suele reprocharse al paradigma, tanto desde posiciones críticas como afines, es la atención, hasta cierto punto limitada, que se ha prestado a las restricciones que impone la actual dinámica de producción de noticias (Hackett, 2006; Keeble, 2010).

Ciertamente, el periodista no es un sujeto aislado, su tarea se desarrolla en un determinado contexto institucional, nacional e internacional no exento de obstáculos. Entre ellos, podemos mencionar: la falta de mecanismos efectivos de autorre- 
gulación y seguimiento de los códigos éticos o sanción en casos de incumplimiento (Tehranian, 2002); las consecuencias de la concentración de la propiedad y de los procesos de "financiarización" en la pluralidad de estructuras y de contenidos (Almirón, 2010); o la inmersión de los propios medios de comunicación y de los Estados en unas relaciones de desigualdad, en la línea de las argumentaciones planteadas en el ya clásico Informe McBride (Hackett, 2006).

En el análisis del conjunto de limitaciones estructurales al periodismo de paz resulta esclarecedor, pese a los años que han pasado desde que fuera formulado, el Modelo de Propaganda de Chomsky y Herman. En su obra Manufacturing consent (1988) (traducida al castellano como Los guardianes de la libertad, 1990), estos autores identifican un sistema de cinco filtros o presiones institucionalizadas que delimitan la acción de los medios y los hace funcionales a los intereses de las elites. Estos cinco mecanismos, que interactúan y se refuerzan entre sí, conforman una especie de tamices a través de los cuales pasa la información, determinando lo que es "digno de publicarse" (Chomsky y Herman, 1990: 22). Dichos filtros son: 1) los intereses corporativos y comerciales, incluyendo el grado de concentración, riqueza y tamaño de la propiedad de los medios; 2) la dependencia de la publicidad como principal fuente ingresos; 3) el recurso a la información procedente del gobierno, empresas y expertos como fuentes habituales; 4) los métodos para acallar los contenidos críticos en los medios; y 5) el "anti..." como mecanismo de control ideológico, la creación del enemigo. Concretamente, Chomsky y Herman hablan en su obra del "anticomunismo", estrategia ideológica que, en la actualidad, podríamos decir que ha sido suplantada, por ejemplo, por el antiterrorismo. En definitiva, el intento de implantar un nuevo paradigma periodístico que promueva el cambio social bajo la influencia de este modelo es, tal y como resume Becker, una tarea prácticamente imposible:

Si los procesos de producción y recepción mediática son, al mismo tiempo, expresión y motor de la violencia estructural; si las tecnologías de la comunicación pueden ser entendidas, históricamente, sólo como una parte integral del complejo militar industrial emergente; si el acceso a los medios y el poder sobre los mismos es desigual y desequilibrado... entonces la expectativa de que los medios de comunicación puedan cumplir con su función de 'portadores de la paz' [sólo será posible] bajo extrañas y excepcionales circunstancias (1982: 227).

Sin embargo, algunos autores consideran excesivamente determinista el enunciado original del Modelo de Propaganda. En este sentido, plantean que no es tan clara la unanimidad y homogeneidad en los discursos mediáticos y que existe cierto margen de agencia para los periodistas en el seno de los medios de comunicación tradicionales (Shoemaker y Reese, 1996; Kempf, 2007b). En palabras de Hackett, el Modelo de Propaganda "tiende al reduccionismo, simplifica de manera excesiva la complejidad del sistema mediático, dice muy poco acerca de los periodistas y de cómo las audiencias interpretan la información" (2006: 4). Es así como es posible encontrar, transmitidos por los medios de comunicación dominantes, notables ejemplos de textos e informaciones críticas con el sistema y basadas en otro tipo de valores.

Ahora bien, a pesar de estas críticas que invitan a una reformulación del Modelo de Propagada, las dificultades para implementar el periodismo de paz en el marco de 
los medios de comunicación dominantes son, sin duda, considerables (Tehranian, 2002: 74). Por ello, autores como Hackett (2010) apuestan por analizar las posibles sinergias entre el periodismo de paz y otros proyectos que, directamente, centran su atención en la necesidad de implementar cambios estructurales en el control y propiedad de los medios. De esta forma, se abre el debate en torno a la complementariedad entre el periodismo de paz e iniciativas como el periodismo alternativo o los movimientos por la democratización del sistema mediático.

En definitiva, el análisis sistemático de los obstáculos y limitaciones en el desarrollo del periodismo de paz, como nuevo paradigma, es todavía una tarea relativamente pendiente. Y, sin embargo, conocer el complejo entramado de relaciones y procesos que finalmente derivan en un determinado producto informativo es fundamental si lo que se pretende es incidir sobre ese producto concreto, especialmente cuando éste aborda realidades marcadas por el conflicto. Así pues, podemos concluir con Hackett que, "para tener éxito, el periodismo de paz debe traducir sus preocupaciones normativas, arraigadas en la disciplina de la investigación para la paz, en una estrategia basada en el análisis fundamentado teóricamente sobre las lógicas que determinan la producción de noticias" (2006: 2). De esta forma, se requiere una profundización de la investigación tanto empírica como teórica así como la combinación del enfoque individual dominante del paradigma (centrado en la agencia del periodista) con un enfoque estructural y global que permita considerar los condicionantes institucionales, nacionales e internacionales realmente existentes.

\section{CONCLUSIONES}

El periodismo de paz es un paradigma relativamente nuevo cuyas características definitorias, en particular las que rodean su nacimiento y desarrollo inicial, van a determinar su posterior evolución. El hecho de que las primeras formulaciones procedan del campo de la investigación para la paz, plasmadas en los planteamientos de Galtung y de veteranos periodistas dedicados, tras décadas de actividad profesional, al mundo del activismo y la docencia e investigación, suponen que el modelo responda, principalmente, a toda una serie de preocupaciones de carácter ético y normativo, por un lado; y a estrategias de acción concretas para mejorar de manera práctica la tarea de informar sobre conflictos, por el otro.

El periodismo de paz nace, por tanto, como una propuesta clara de movilización y acción comunicativa para el cambio social. Si bien es cierto que no tarda mucho en captar el interés de algunos investigadores de otros campos, cabe resaltar que los primeros trabajos académicos seguirán también esta misma tendencia: "A pesar de que existe un conjunto excelente de literatura e investigación sobre el periodismo de guerra [...], la mayor parte del trabajo sobre periodismo de paz es normativo o prescriptivo, destacando sus beneficios y detallando cómo puede ser implementado" (Lee y Maslog, 2005: 313). Esta circunstancia permite identificar una serie de lagunas en materia de investigación, especialmente en lo que respecta al desarrollo de indicadores empíricos y al análisis contextual (mediático, político y social) en que 
se enmarca. De algún modo, el periodismo de paz salta de la teoría a la práctica adoleciendo de los beneficios de la investigación empírica (Lee y Maslog, 2005).

Éste es, precisamente, uno de los aspectos en los que el periodismo de paz ha de concentrar sus esfuerzos: la operacionalización de sus supuestos normativos. Aquello por lo que el periodismo de paz es deseable desde un punto de vista ético debe transformarse, según Shinar y Kempf, "en una estrategia basada en el análisis teórico y práctico de las lógicas y los límites de la producción de noticias" (2007: 4). Los análisis o estudios de caso que se han realizado hasta la fecha pueden ser un buen punto de partida para redefinir sus indicadores empíricos. Avanzar en el desarrollo de un marco metodológico riguroso y realizar nuevos estudios de caso específicos, enfocados sobre todo en el análisis de las coberturas poniendo un énfasis especial no tanto en el periodismo de guerra sino en el periodismo de paz, "puede ayudarnos a especificar las condiciones bajo las cuales los medios de comunicación podrían jugar un papel más constructivo en cuanto a la resolución de los conflictos" (Hackett, 2007:50).

Tal y como hemos visto, el periodismo de paz se enfrenta a obstáculos de índole cultural y estructural, conectados con el funcionamiento mismo de la sociedad y sus características sistémicas. En este sentido, el paradigma dominante o periodismo de guerra no es más que un reflejo de una sistema social que genera violencia en sí mismo y del cual los medios vienen a ser un elemento más a la hora de reproducir esas relaciones sistémicamente violentas (Becker, 1982). Sería ingenuo, por tanto, considerar que el éxito del modelo depende únicamente de introducir algunas reformas en las rutinas profesionales o en la formación de los periodistas. Éstas son obviamente necesarias, sin embargo, el periodismo de paz como paradigma va mucho más allá de lo meramente informativo. Su potencial transformador reside en la capacidad para incorporar cuestiones fundamentales sobre el funcionamiento mismo de la sociedad, la relación de ésta con los medios de comunicación y cómo la actual cobertura de los conflictos refleja un modo particular de entenderlos, abordarlos y resolverlos. Ahí radica precisamente la paradoja del modelo, como bien apunta Hackett (2007), en que su viabilidad depende en gran medida de las condiciones que trata de promover; en definitiva, una comunicación más democrática en la que tengan cabida todas las voces y que fomente otro tipo de valores. La idea, en resumen, es clara: la evolución del periodismo hacia los postulados del periodismo de paz sólo podrá ser efectiva de manera integral en un mundo que avance a su vez hacia formas de relación y de resolución de los conflictos más pacíficas y no-violentas. 


\section{REFERENCIAS BIBLOGRÁFICAS}

Almiron, N. (2010): Journalism in Crisis. Corporate Media and Financialization,Cresskill NJ: Hampton Press.

BECKER, J. (1982): "Communication and peace. The empirical and theoretical relation between two categories in social sciences", Journal of Peace Research 19 (3), 227-240.

Chomsky, N. y Herman, E.S. (1990): Los guardianes de la libertad. Barcelona: Editorial Crítica.

CURRAn, J. (2005): Medios de comunicación y poder en una sociedad democrática. Barcelona: Hacer.

FAwCETt, L. (2002): “Why Peace Journalism isn't news?”. Journalism Studies, 3 (2): 213-223.

FISAS, V. (1998): Cultura de paz y gestión de conflictos. Barcelona: Icaria.

FrÈre, M. S. (2007): The media and conflicts in Central Africa. Londres: Lynne Rienner Publishers.

Galtung, J. (1990): “Cultural violence”, Journal of Peace Research, 27(3): 291-305.

(1998a): "High road, low road: charting the course for Peace Journalism", Track Two, 7(4): Disponible: http://ccrweb.ccr.uct.ac.za/archive/two/7_4/index74.html[consultado el 24 de febrero de 2012]

- (1998b): Tras la violencia, 3R :reconstrucción, reconciliación, resolución: afrontando los efectos visibles e invisibles de la guerra y la violencia. Bilbao: Bakeaz.

(2000): "The task of Peace Journalism", Ethical Perspectives, 7 (2-3): 162-164.

(2002): "Peace journalism -A Challenge" en K. Wilhem y H. Luostarinen (eds.), Journalism and the New World Order. Göteborg: Nordicom, pp. 259-272.

- (2006): "Peace Journalism as an Ethical Challenge", Global Media Journal: Mediterranean Edition 1(2): 1-5.

- (2007): "Peace Journalism as Ethical Challenge", Asteriskos, 3/4: 7-16.

Galtung, J.; Lynch, J. y McGoldrick, A. (2006): Reporteando conflictos. Una introducción al periodismo de paz. Puebla: Montiel \& Soriano Editores.

Galtung, J. y Ruge, M. H. (1965): "The Structure of Foreign News. The Presentation of the Congo, Cuba, and Cyprus Crises in Four Norwegian Newspapers", Journal of Peace Research, 2(1): 64-91.

Galtung, J. y Vincent, R. C. (1995): Global Glasnost. Towards a New World Information and Communication Order?. Cresskill, NJ: Hampton Press.

GIRÓ, X. (2004): "Esbotzada de barreres en la cobertura de la Guerra a l'Iraq", Quaderns del $C A C$, número extraordinario, septiembre: 179-187.

(2007): "Enfoques analíticos críticos sobre el discurso de la cobertura informativa de conflictos" en T. Telleschi y E.A. Sandoval (coords.) Espacio y tiempo en la globalización. Una visión de la transparencia en la información. Toluca, México: Universidad de Pisa y Comisión Estatal para el Acceso a la Información Pública del Estado de Sinaloa: 199-220.

Giró, X. y JARQUe, J.M. (2006): "Prensa escrita e inmigración: estudio sobre la opinión de los diarios sobre la inmigración procedente de fuera de la Unión Europea y sobre la cobertura informativa de conflictos destacados que tienen relación con ella (octubre 1999-junio 2002)", ZER, 20: 251-270.

HACKet, R. A. (2006): "Is Peace Journalism possible? Three frameworks for assessing structure and agency in news media", Conflict \& communication online, 5 (2).

- (2007): "Journalism versus Peace? Notes on a problematic relationship", Global Media Journal: Mediterranean Edition, 2 (1): 47-53. 
(2010): "Journalism for Peace and Justice: Towards a Comparative Analysis of Media Paradigms", Studies in Social Justice, 4 (2): 179-198.

Hackett, R.A. y Schroeder, B. ( 2008): "Does Anybody Practice Peace Journalism? A Cross-National Comparison of Press Coverage of the Afghanistan and Israeli-Hezbollah Wars", Peace and Policy, 13(1): 26-47.

Hackett, R.A. y Zhao, Y. (1998): Sustaining democracy? Journalism and the politics of objectivity. Toronto: Garamond.

IEP (2010): Measuring peace in the media. Special Report. Disponible en: http://www.visionofhumanity.org/info-center/measuring-peace-in-the-media/ [Consultado el 24 de febrero de 2012]

IRVAN, S. (2006): "Peace Journalism as a normative theory: premises and obstacles". Global Media Journal: Mediterranean Edition, 1 (2), pp.34-39.

KeEBLE, R. L. (2010): "Peace Journalism as political practice: a new, radical look at the theory", en R.L. Keeble, J. Tulloch y F. Zollmann (eds.), Peace Journalism, war and conflict resolution. Nueva York: Peter Lang, pp. 49-68.

KEMPF, W. (2007a): "Peace Journalism: a tightrope walk between advocacy journalism and constructive conflict coverage", Conflict \& communication online, 6 (2).

- (2007b): "Two experiments focusing on de-escalation oriented coverage of post-war conflicts”, en D. Shinar y W. Kempf (eds.), Peace journalism: The state of the art. Berlin: Verlag Irena Regener, pp. 136-157.

LEDEREACH, J. P. (2000): El abecé de la paz y los conflictos. Eduación para la paz. Madrid: Los libros de la catarata.

LeE, S. T. y Maslog C. (2005): "War or Peace Journalism? Asian newspaper coverage of conflicts", Journal of Communication, 55 (2), pp. 311-329.

LYNCH, J. (2008): Debates in peace journalism. Sydney: Sydney University Press. (2009): “Active and passive peace journalism in reporting of the 'war on terrorism' in the Philippines" en S.D. Ross y M. Tehranian, Peace journalism in times of war, Nueva Jersey: Transaction Publishers, pp. 143-162.

LYNCH, J. y McGoldRICK, A. (2005): Peace Journalism, Londres: Hawthorn Pres.

MAsloG, C.; LeE, S.T. y Kim, H. S. (2006): "Framing analysis of a conflict: how newspapers in five Asian countries covered the Iraq War". Asian Journal of Communication, 16 (2), pp.19-39.

NASSANGA, L. (2007): "Peace Journalism applied: an assessment of media coverage of the conflict in Northern Uganda", Conflict \& communication online, 6 (2).

Ottosen, R. (2007): "Emphasising images in Peace Journalism: theory and practice in the case of Norway's biggest newspaper", Conflict \& communication online, 6 (1).

Peleg, S. (2006): "Peace Journalism through the lense of conflict theory: analysis and practice", Conflict \& communication online, 5 (2).

(2007): "In defense of peace journalism: a rejoinder", Conflict \& communication online, 6 (2).

Penalva, C. (2002): "El tratamiento de la violencia en los medios de comunicación", Alternativas. Cuadernos de Trabajo Social, 10: pp. 395-412.

Ross, S. D. (2008): “A summer's pastime: strategic construction of the 2006 war in Lebanon". Peace and Policy, 13: pp.47-61

SHINAR, D. (2009): "Why not more peace journalism? The coverage of the 2006 Lebanon war in Canadian and Israeli media", en S.D. Ross y M.Tehranian, Peace journalism in times of war, Nueva Jersey: Transaction Publishers, pp. 7-17.

Shinar, D. Y Kempf, W. (eds.) (2007): Peace Journalism: The State of the Art. Berlin: Regener. 
Shoemaker, P. y ReEse, S. (1996): Mediating the message: Theories of influences on mass media content. White Plains, NY: Longman.

Tehranian, M. (2002): "Peace Journalism. Negotiating global media ethics", The Harvard International Journal of Press/Politics, 7 (2): 58-83.

Thompson, A. (ed.) (2007):The media and the Rwanda Genocide. Londres: Pluto Press.

Tortosa, J. M. (2003): "Los medios y la guerra", Revista de Estudios Sociales, 6: 57-69.

\title{
RESUMEN
}

El periodismo de paz constituye un paradigma orientado al cambio social cuyo principal objetivo es dotar a los profesionales de la comunicación de herramientas analíticas y prácticas que les permitan abordar el conflicto de manera constructiva y éticamente responsable. Supone un desafío a la forma de interpretar los propios conflictos, las relaciones entre medios de comunicación y sociedad y el rol que los periodistas pueden o deben jugar en contextos de esta naturaleza. En el presente artículo se plantea una revisión teórica del periodismo de paz que nos va a permitir reformularlo conceptualmente, delimitar sus dimensiones y establecer los obstáculos o límites que, dadas las características del sistema mediático hegemónico, dificultan hoy en día su éxito como modelo de comunicación, clave en la construcción de una cultura de paz.

Palabras clave: periodismo de paz, conflicto, paz, violencia, comunicación, cambio social.

\begin{abstract}
Peace journalism is a relatively new paradigm oriented to social change. Its main objective is to equip communication professionals with the analytical and practical tools required to report constructively and ethically about conflicts and wars. But it is also a challenge for understanding conflicts, the relationship between media and society and the role journalists can or must play in conflict situations. The aim of this article is to develop a theoretical review of peace journalism related to definitions, characteristics, concepts and dimensions. This revision will also allow as to point out the barriers for peace journalism success as a model of alternative communication for the construction of a culture of peace.
\end{abstract}

Key words: peace journalism, conflict, peace, violence, communication, social change.

\section{RÉSUMÉ}

Le journalisme de paix est un paradigme relativement nouveau, orienté au changement social. Son but principal est celui de préparer les professionnels de la communication avec les instruments analytiques et pratiques pour faire un journalisme constructif et éthique dans les conflits et les guerres. Mais il s'agit aussi du défi de la compréhension des conflits, de la relation des médias et la société et du rôle des journalistes dans les situations conflictuels. Le but de cet article est celui de développer une révision théorétique du journalisme de paix en relation avec les définitions, les traits, les concepts et les dimensions. Cette révision nous permettra aussi marquer les barrières au journalisme de paix comme modèle de communication alternative pour la construction d'une culture de paix.

Mots clé: journalisme de paix, conflit, paix, violence, communication, changement social. 